\title{
GLOBAL IMAGE CONTRAST ENHANCEMENT ALGORITHM USING HISTOGRAM-MODIFICATION FRAMEWORK
}

\author{
P. Swathi ${ }^{1}$, I. Jithendra ${ }^{2}$, L. Guru Kumar ${ }^{3}$ \\ ${ }^{1}$ M.Tech. (D.E.C.S.), N.B.K.R. Institute of Science \& Technology, Vidyanagar, Nellore (D.t.), Andhra Pradesh- \\ 524413, India \\ ${ }^{2}$ M.Tech. (D.E.C.S.), N.B.K.R. Institute of Science \& Technology, Vidyanagar, Nellore (D.t.), Andhra Pradesh- \\ 524413, India \\ ${ }^{3}$ Asst. Professor, Dept. of E.C.E, N.B.K.R. Institute of Science \& Technology, Vidyanagar, Nellore (D.t.), Andhra \\ Pradesh-524413, India
}

\begin{abstract}
In this paper, we tend to propose a worldwide substitution qualification change algorithmic project misuse based on the histograms of shading and profundity pictures. On the thought about the histogram-change framework, the shading and significance picture histograms area unit[1] begins parcel into sub intervals abusing the Gaussian mix model. The positions apportioning the shading histograms territory unit acclimated to spatially neighbor pixels with an identical power and profundity qualities will be sorted into a proportionate interim. By assessing the mapping bend of the refinement change for each interim, misuse this model worldwide refinement change will be enhanced while not over-improving the local picture capability. Test outcomes demonstrate the ampleness of the anticipated algorithmic system.
\end{abstract}

Keywords - Contrast enhancement, depth image, histogram modification, histogram partitioning

\section{INTRODUCTION}

Histogram processing is the demonstration of modifying a picture by altering its histogram. Regular employments of histogram handling incorporate standardization by which one makes the histogram of a picture as level as could be allowed. In PC design, the procedure of enhancing the nature of a digitally put away picture by controlling the picture with programming. It is simple, for instance, to make a picture lighter or darker, or to expand or lessening differentiation. Propelled picture improvement programming additionally bolsters numerous channels for adjusting pictures in different ways. Projects specific for picture improvement[2] are some of the time called picture editors Image upgrade is the procedure of altering computerized pictures so that the outcomes are more suitable for showcase or further picture examination. The point of picture improvement is to enhance the interpretability or impression of data in pictures for human viewers, or to give 'better' information for other computerized picture preparing systems. An exceptionally well known strategy for difference upgrade of pictures is Histogram Equalization (HE).

i. Image Enhancement

ii. Image Restoration

iii. Image Compression etc.

The rest of the paper is organized as follows. In Section II, Whatever is left of the paper is composed as takes after. In Segment II, histogram out for picture contrast improvement calculation is depicted. Proposed algorithm steps Clarified in Area III, Test results are displayed in Segment IV, trailed by the conclusion in Segment V.

\section{HISTOGRAM EQUALIZATION}

This technique typically increases the global contrast of many pictures, particularly when the usable data of the image is represented by near contrast values. Through this modification, the intensities are often higher distributed on the bar chart. This permits for areas of lower native distinction to realize a better distinction. HE accomplishes this by effectively spreading out the foremost frequent intensity values. This technique is beneficial in footage with backgrounds and foregrounds that area unit each dark or each bright. In particularly, the method will result in higher views of bone structure in $\mathrm{x}$-ray pictures, and to raised detail in pictures that area unit over or under-exposed. A key advantage of the strategy is that it's a reasonably simple technique and an invertible operator[3]. Therefore during this theory, if the HE operate is understood, then the initial bar chart are often recovered. The calculation isn't computationally intensive. A downside of the strategy is that it's indiscriminate. It's going to increase the distinction of background signal, whereas decreasing the usable signal. In scientific imaging wherever space al correlation is additional vital than intensity of signal (such as separating DNA fragments[4] of quantal length), the little signal to noise magnitude relation sometimes hampers visual detection. HE often produces unrealistic effects in photographs; then again it is extremely helpful for investigative pictures like warm, satellite or $\mathrm{x}$ beam pictures, frequently the same class of pictures that client would apply false-shading to. Additionally histogram evening out can deliver undesirable impacts (like unmistakable picture angle) when connected to pictures with low shading profundity. 
The cutting edge calculation models the picture histogram utilizing the Gaussian Mixture Model (GMM) and partitions the histogram utilizing the crossing point purposes of the Gaussian parts. The separated sub-histograms are then autonomously augmented using assessed Gaussian parameters. As of late, specialized leaps forward of the shading picture upgrade have been discovered utilizing profundity or stereo as side data. Stereo coordinating calculations and profundity sensors are presently giving very precise profundity pictures, and in this manner the utilization of the profundity picture for the shading picture improvement turns into a vital exploration issue. In this paper we propose another difference upgrade calculation that adventures the histograms of both shading and profundity images[4][5]. The histograms of shading and profundity pictures are initially separated into sub-interims utilizing the GMM. The interims of the shading picture histogram are then balanced such that the pixels with the comparable power and profundity qualities can fit in with the same interim. The proposed calculation is hence implicitly depth versatile, and the test results show the adequacy of the proposed calculation.

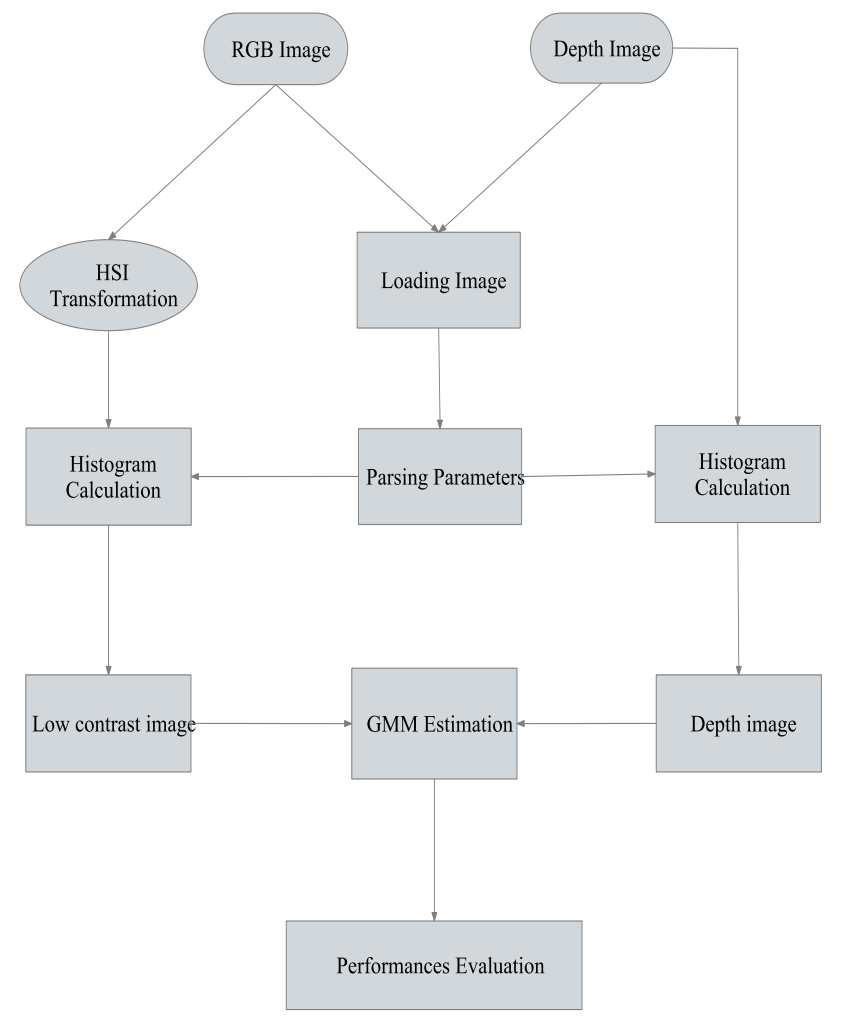

Algorithm

\section{MODULE DESCRIPTION}

\subsection{Depth Image Generation}

Depth images are obtained by stereo vision method. The 3Ddata may be obtained from a try of images, additionally called a stereo try, by evaluating the relative profundity of focuses insides of the scene. These estimation region unit delineated in an extremely stereo disparity map, that is built by matching corresponding points within the stereo try.
Color depth, additionally called bit depth, is either the amount of bits wont to indicate the color of one component, in a very bitmapped images or video buffer store, or the amount of bits used for every color element of one component[6]. For client video standards, like high potency video writing the bit depth specifies the amount of nits used for every color element. Once relating a component the idea may be outlined as bits per component that species the amount of bits used. Once relating a color element the idea may be outlined as bits per channel bits per color or bits per sample. color depth is just one side of outline illustration, expressing however levels of color may be expressed the opposite side is however board a spread of color may be the definition of each color preciseness and gamut is accomplished with a color cryptography specification that assigns a digital code worth to a location in a very color house.

\subsection{HSI Separation}

The Hue, Saturation and Intensity qualities were divided from the RGB picture. Hue is the extent to which a shading can be portrayed as like or not quite the same as shading that are depicted as red, green, blue, and yellow. Saturation is the level of contrast between a shading and dark. Intensity is the shine or bluntness of hues. Intensity is the measure of measure of light force saw by eye paying little respect to the color. HSL are the most widely recognized barrel shaped direction representations of focuses in a RGB shading model[7]. Grown in the 1970s for PC design applications, HSL are utilized today as a part of shading pickers, in picture altering programming, and less generally in picture examination and PC vision.

The two representations improve the geometry of RGB trying to be more instinctive and perceptually pertinent than the Cartesian (3D shape) representation, by mapping the qualities into a barrel approximately motivated by a customary shading wheel. The edge around the focal vertical pivot compares to "tone" and the separation from the hub relates to "immersion". These initial two qualities give the two plans the " $\mathrm{H}$ " and "S" in their names. The stature relates to a third esteem, the framework's representation of the apparent luminance in connection to the immersion. Seen luminance is a famously troublesome part of shading to speak to in a computerized configuration (see impediments area), and this has offered ascent to two frameworks endeavoring to comprehend this issue: HSL (L for daintiness) and HSV or HSB (V for quality or B for shine). A third model, HSI (I for power), normal in PC vision applications, endeavors to adjust the favorable circumstances and drawbacks of the other two frameworks[8]. While commonly reliable, these definitions are not institutionalized, and the shortened forms are casually tradable for any of these three or a few other related round and hollow models. Note likewise that while "shade" in HIS alludes to the same property, their meanings of "immersion" contrast significantly. (For specialized meanings of these terms, see Color-production traits.) 
Both of these representations are utilized broadly as a part of PC illustrations, yet both are likewise censured for not satisfactorily differentiating shading making characteristics, and for their absence of perceptual consistency. This implies that the shading showed on one screen for a given HSV worth is unrealistic to precisely coordinate the shading seen on another screen unless the two are definitely changed in accordance with outright shading spaces.

\subsection{Histogram Calculation}

Histogram is the graphical representation of the tonal distribution of the image. The histogram values are calculated for input and depth images. The histogram values were compared and the positions where the histogram values were changing is identified and the points were located.

Contrast improvement techniques[9] within the second subgroup modify the images through some component mapping specified the bar graph of the processed images is additional unfold than of the first image. Techniques during this subgroup either enhance the distinction globally or domestically. IFA single mapping derived from the images is employed then it's a world methodology; if the neighborhood of every component id employing to get $s$ neighborhood mapping perform then it's a neighborhood method. Employing a single world mapping can't enhance the native distinction

\subsection{GMM Estimation}

A GMM may be a natural model to use if a category contains variety of distinct subclasses, as is usually the case. Another to GMMs would then appears to be to treat the elements of the GMMs for every block as hidden states and to couples them to every different sportily, so manufacturing a $2^{\text {nd }}$ GNN. Whereas this might sound sort of a sensible plan, the parameters estimation drawbacks becomes a great deal of harder, each algorithmically and since there square measure additional parameters to be calculated form an equivalent restricted quantity of information[10]. Additionally, since the linguistics of the hidden states may be a prior unknown, it's not clear that the dependencies introduced by the GMM square measure blessing. We choose to receive the less confused methodology, to be confirmed a posterior by comparison with the GMM different as used, as an example, by Li and gray.

\subsection{Color Enhancement}

Mapping Curve[11][12] of Color Intensity is obtained by scrutiny the calculable bar graph of original and therefore the depth image. The region that must be changed is known and that they square measure changed. The ultimate result's the distinction enhance color image. The principal objective of image sweetening is to method a given image so the results additional appropriate than the main picture for a chose application. It emphasizes or hones picture alternatives[13] like edges, limits, or distinction to create a graphic show additional useful for show more, examination.
The sweetening does not assemble the inherent information substance of the information, on the other hand it will expand the element fluctuate of the picked[14] choices so they'll be distinguished basically.

Low-contrast pictures may result from poor illumination, lack of dynamic point the image device, or perhaps wrong setting of a lens aperture throughout image acquisition. The concept behind distinction[15] stretching is to extend the dynamic vary of the grey levels within the image being processed. It's typically necessary to reinforce details[16] [17] over little areas. The quantity of pixels in these areas might have negligible influence on the computation of a worldwide transformation, that the use of worldwide bar graph specification doesn't essentially guarantee the specified native sweetening.

\section{EXPERIMENTAL RESULTS}

In order to judge the performance of the planned formula, the Middlebury stereo check pictures were utilized in our experiment. The depth pictures were obtained victimization the stereo matching formula as shown in Fig. 3.
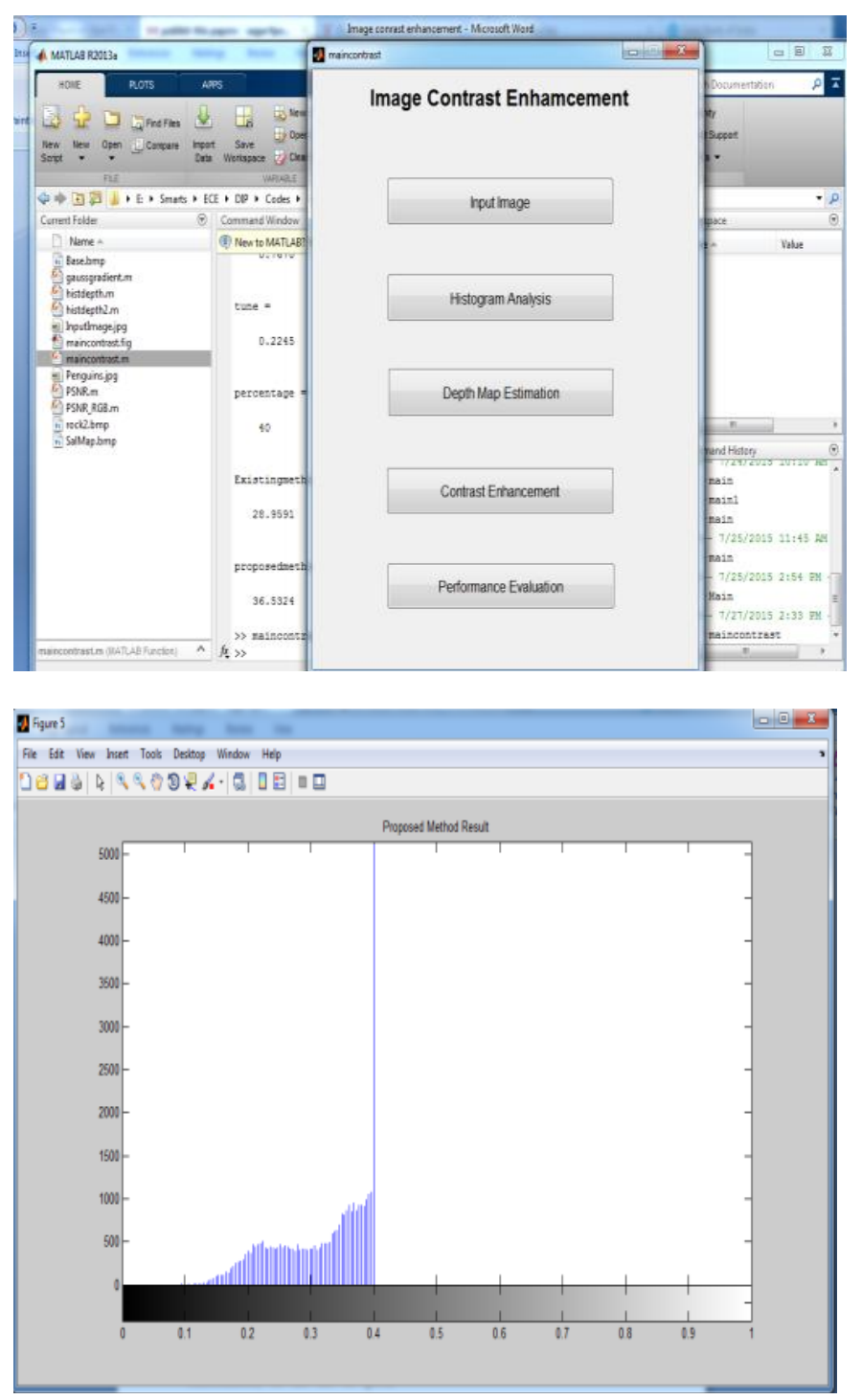

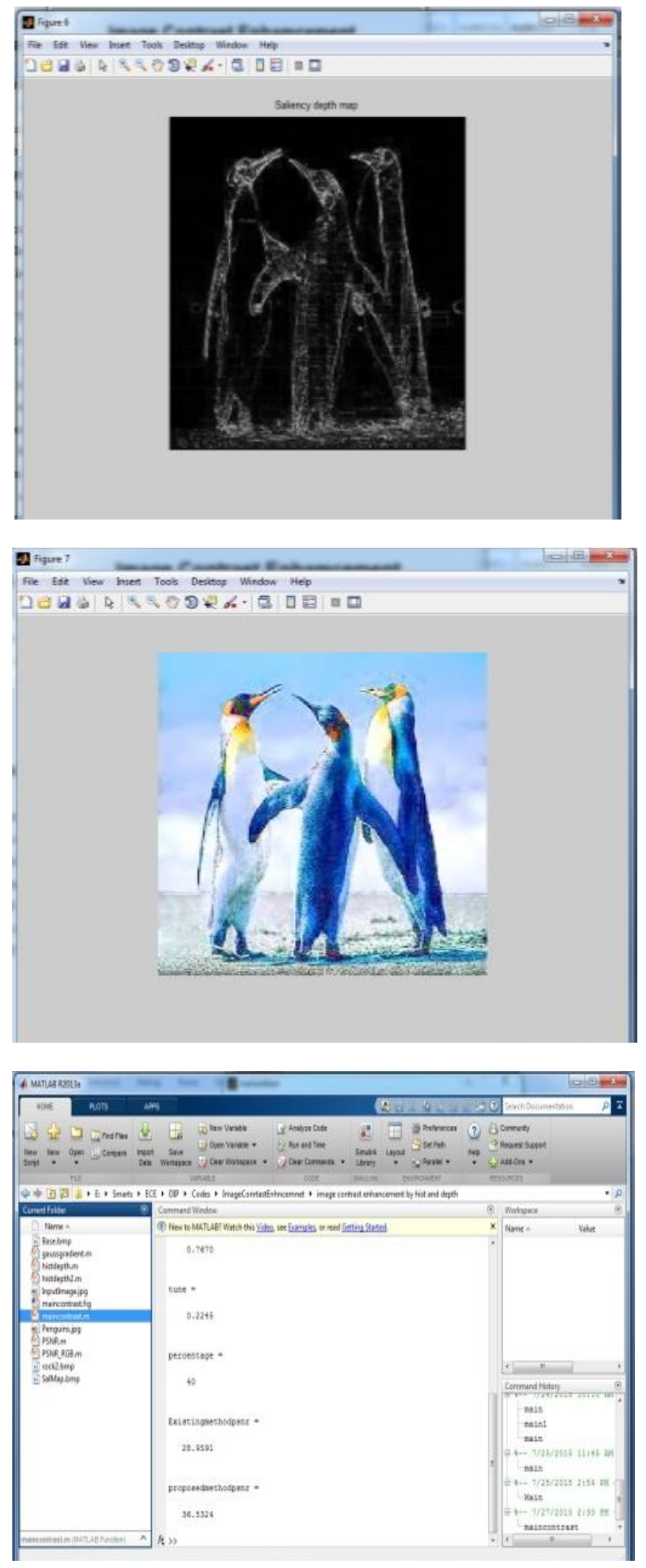

\section{CONCLUSION}

In this paper we have a tendency to plan a brand new histogram-based image distinction sweetening formula victimization the histograms of color and depth pictures. The histograms of the shading and profundity pictures square measure first partitioning into sub-interims exploitation the GMM. The divided histograms square measure then accustomed get the layer labeling results of the color and depth pictures. The sub-intervals of the color bar graph square measure adjusted specified the pixels with identical intensity and depth values will belong to identical layer. Therefore, whereas a worldwide image distinction is stretched, an area image distinction is additionally systematically improved while not the over-enhancement. They arrange to extend our layer-based formula to a segment-based formula by employing a joint color-depth segmentation methodology.

It doesn't introduce a flicker. This is often chiefly thanks to the very fact that the strategy uses the input bar graph, that doesn't modification considerably inside identical scene, because the primary supply of knowledge. This methodology is applicable to a large form of pictures. It additionally offers level of controllability and adaptableness through that completely different levels of distinction sweetening, from bar graph leveling to no distinction sweetening, may be achieved.

\section{REFERENCES}

[1] Salih Dikbas, Senior Member, IEEE “ A bar chart modification framework and its application for image distinction upgrade," IEEE Trans. Image method., Vol. 18, no. 9, pp. 1921-1935, Sep. 2009 .

[2] S. Choi, J. Lu, B.Ham, "profundity feature upgrade taking under consideration weighted mode separation," IEEE Trans. Image method, vol. 21, no. 3, pp. 1176-1190, Mar.2012

[3] M. M. Subedar and L. J Karan, "Expanded profundity recognition with sharpness Upgrade for stereo feature," in Proc. SPIE, 2010, Vol.7524, p.75241B

[4] K. Lai, L. Bo, X. Ren,” A Vast scale progressive multiview RGB-D object dataset," in Proc. IEEE Int. Conf. Apply autonomy and Automation (ICRA),2011, pp. 1817-18

[5] M. Kaaniche, Faouzi Alaya Cheikh, "consolidating profundity knowledge and near edge recognition for stereo image upgrade," in Proc. Eur. signal process. Conf. (EUSIPCO), 2012, no. 1, pp. 250-254.

[6] S. W. Jung. "Improvement of image and profundity guide utilizing adapative joint trilateral channel," IEEE Trans. Circuits Syst. Feature Techo., 21, no. 3, pp. 1191-1199 Mar. 2012

[7] M. M. Subedar and L. J. Karam, "Expanded profundity recognition with sharpness Upgrade for stereo feature," in Proc. SPIE, 7524 stereoscopic Displays Appl. XXI, 2010, p. 1-8.

[8] Q.Yang," A non-nearby price aggregation method for stereo coordinative," in Proc. IEEE Comput. Vis. Style Recogn., 2012, pp. 1402-1409.

[9] M. Figueredo and A. Jain, "unsupervised learning of restricter mixture models," IEEE Trans. Patt. Anal. Mach. Intell., vol. 24, no. 3, pp. 381-396, Mar. 2002.

[10] Wa James Tam, J Flack, "Smoothing profundity maps for increased photograph quality," Proc. Int. 
Soc. Select. Eng. Conf. three-D TV Feature Show III, pp. 162-172 2004.

[11] Y. K Park, K. Jung, Y. Gracious, "profundity image based mostly rendering for 3-D TV administration over T-DMB," Sign Procedure. Image Commun. Vol. 24, no. 1-2, pp. 122-136 2009.

[12] J. Delon, A. Desolneux, J. L. Lisani, "A nonparametrical approach for bar chart division," IEEE Trans. Image method. vol. 16, no. 253-261, jan. 2007.

[13] S. J. Ko, "Profundity sensation upgrade utilizing the just noticeable profundity distinction," IEEE Trans. Picture no. 8, pp. 3624-3637 Aug. 2012.

[14] H. Y. Shum, S. B. Kang, "Review of image based mostly representations and pressure strategies", IEEE Trans. Circuits Syst. Feature Technol., vol. 13, pp. 1020-1037 2003

[15] C.Fehn "Profundity images based mostly rendering (DIBR), pressure and transmission for one more methodology on 3-D-Television," Proc. SPIE, vol. 5219, no.2, pp. 93-104 2004.

[16] Smolic and D. Mc Cutuhen "3DAV investigation of feature based mostly rendering innovation in MPEG,” IEEE Trans. Circuits Syst. Feature Technol., vol. 14, pp. 348-356 2004.

[17] K. J. Goodness, S. Yea " Gap filling technique utilizing profundity based mostly as an area of painting for perspective combination in free perspective TV and three-D FESTURE, "proc. Images cryptography Symp., pp.1-4.

\section{BIOGRAPGIES}

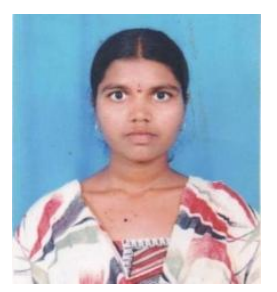

P. Swathi, student of M. Tech., DECS in NBKR Institute of Science \& Technology (Autonomous) received B.Tech. degree from Vikrama Simhapuri University in 2014, Nellore, A.P. Areas of interest are Image Processing, Communication System.

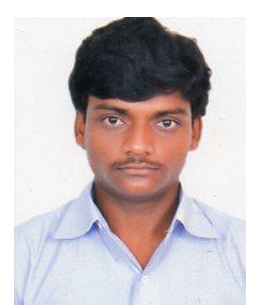

I. Jithendra student of M. Tech., D.E.C.S. in NBKR Institute of Science \& Technology (Autonomous) received B.Tech. degree from Sri Venkateswara University in 2013, Tirupati, A.P. Areas of interest are Image Processing, Communication System.

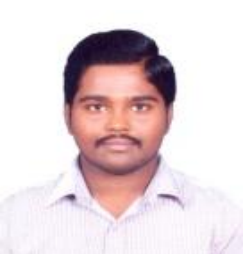

L. Guru Kumar has received M.E. degree from Anna University, T.N in 2012. and B.Tech. degree from Sri Venkateswara University, A.P in 2009. Currently he is working as Assistant Professor in Department of Electronics \& Communication Engineering, N.B.K.R. Institute of Science \& Technology(Autonomous) Estd. 1978. A.P. Signal \& Image Processing, VLSI Design are his areas of interests. 